\title{
Chronic Myeloid Leukemia - Prognostic Value of Mutations
}

\author{
Bushra Kaleem*, Sadaf Shahab, Nuzhat Ahmed, Tahir Sultan Shamsi
}

\begin{abstract}
Chronic myeloid leukemia (CML) is a stem cell disorder characterized by unrestricted proliferation of the myeloid series that occurs due to the BCR-ABL fusion oncogene as a result of reciprocal translocation $t(9 ; 22)$ (q34;q11). This discovery has made this particular domain a target for future efforts to cure CML. Imatinib revolutionized the treatment options for CML and gave encouraging results both in case of safety as well as tolerability profile as compared to agents such as hydroxyurea or busulfan given before Imatinib. However, about 2-4\% of patients show resistance and mutations have been found to be one of the reasons for its development. European Leukemianet gives recommendations for BCR-ABL mutational analysis along with other tyrosine kinase inhibitors (TKIs) that should be administered according to the mutations harbored in a patient. The following overview gives recommendations for monitoring patients on the basis of their mutational status.
\end{abstract}

Keywords: Chronic myeloid leukemia - BCR-ABL mutations - tyrosine kinase inhibitors

Asian Pac J Cancer Prev, 16 (17), 7415-7423

\section{Introduction}

Chronic myeloid leukemia (CML), a malignant haematopoeitic stem cell disease, characterized by the occurrence of the Philadelphia chromosome which is thought to be a definitive diagnostic marker for CML and is present in almost $90 \%$ of the patients (Hagop et al., 2002). This chromosome results due to the balanced reciprocal translocation $\mathrm{t}(9 ; 22)(\mathrm{q} 34 ; \mathrm{q} 11)$. The fusion of the Abelson murine leukemia (ABL) gene on chromosome 9 with the breakpoint cluster region (BCR) gene on chromosome 22 gives rise to the $\mathrm{BCR}-\mathrm{ABL}$ oncogene that encodes an oncoprotein (Saad et al., 2009).

The breakpoints in the BCR gene has been shown to be clustered in three regions, (a) a $5.8 \mathrm{~kb}$ region spanning exons 12-16 (e12-e16), called the major breakpoint cluster region (M-bcr) that codes a $210 \mathrm{kDa}$ chimeric protein (p210), (b) a $55 \mathrm{~kb}$ sequence of the first intron (e1-e2) called the minor breakpoint cluster region (m-bcr) that encodes a $190 \mathrm{kDa}$ chimeric protein (p190) and finally intron 19 , called $\mu$-bcr forming a resultant fusion transcript of $230 \mathrm{kDa}$ protein (p230) (Fausel, 2007; Yuan et al., 2014).

Studies show an association between the genetic modifications within the precursor haematopoietic stem cells that may lead to the development of chronic myeloid leukemia (Meggyesi et al., 2011; Gulzar et al., 2012).

This oncoprotein is an active tyrosine kinase in the $\mathrm{ABL}$ region that promotes unrestricted growth and replication by causing deviations in proliferation and resists apoptosis through multiple downstream path $\neg$ ways, such as Janus kinase signal transducer and activator of transcription (Jak-STAT), MYC etc. (Pavan et al., 2013).
Even after being described for about more than 150 years ago, the steps taken with regards to its treatment were not rewarding enough for a better half of the century. The initial effectual treatment options for chronic myeloid leukemia included radiation therapy to the spleen and conventional chemotherapeutic drugs, mainly Busulfan and Hydroxyurea which helped control the rapid myeloproliferation leading to significantly improved quality of life during the indolent chronic phase (CP) of the disease. However, it did not have any important role in either preventing or delaying the progression towards accelerated phase or the blast crisis and thus had a limited effect on overall survival (OS) (Michele et al., 2006; Hehlmann et al., 2007; Bjorkholm et al., 2011; Kantarjian et al., 2012).

The introduction of allogeneic stem cell transplantation became the first major step forward in terms of treatment of CML, as about $50 \%$ of the patients who were eligible for alloSCT were cured as proven by them being both BCR-ABL negative as well as Philadelphia negative after the transplant. However the drawback in case of the transplantation was that it gave the best results in patients who were less than 40 years old, while CML being a disease of the elderly had median age at diagnosis of 60 years. The development of chronic graft-versus-hostdisease was another major hurdle in this treatment option (Michele et al., 2006; Hehlmann et al., 2007; Baccarani et al., 2009; Pavlu et al., 2011; Baccarani et al., 2013).

The second major success came in the form of human recombinant interferon-alfa ( $\mathrm{rIFN} \alpha)$, which gave complete cytogenetic response (CCyR) in $15 \%$ to $30 \%$ of patients, and also provided with much better survival benefit over conventional chemotherapy (Hagop et al., 2010; Pavan et 
al., 2013; Talpaz et al., 2013).

The advent of imatinib mesylate (IM) in the late 90 's created a revolution in terms of the treatment of patients with chronic myeloid leukemia (Hehlmann et al., 2007; Thomas et al., 2009). Imatinib Mesylate (IM) the selective inhibitor of the BCR-ABL tyrosine kinase, competitively targets the adenosine 5'-triphosphate binding site of the Abl Kinase Domain and thus blocks downstream signal transduction pathways leading to growth arrest and apoptosis (Hagop et al., 2010; Yasser et al., 2013). Imatinib has become the standard treatment for CML as it is extremely safe with minimal side-effects. A 7-year follow-up of the International randomized study of interferon versus STI571 (IRIS) study showed that the overall survival (OS) in CML patients who received IM as the first line drug was $86 \%$ and the progression to advanced stage of the disease (i.e. either the accelerated or the blastic phase) was reduced by $93 \%$ (Stephen et al., 2010).

However, a small proportion of patients in the chronic phase of the disease and significant number of patients in the advanced phase showed refractoriness to the treatment either in the initial stage or acquired resistance after initial response, thus resulting in relapse (Hochhaus et al., 2007; Thomas et al., 2009). Of all the reasons for the acquired resistance to occur, most common cause is the emergence of point mutations within the BCR-ABL kinase domain which leads to an impairment in the Imatinib binding by creating an obstacle at the site where the Imatinib or by preserving the configuration of BCR-ABL which has reduced Imatinib binding affinity (O'Hare T et al., 2007). Analysis of CML patients found to be resistant to Imatinib had the presence of more than 90 different BCRABL kinase domain mutations that encoded more than 50 different amino acid substitutions, although some are definitely more frequent than others. Different mutations lead to variable degrees of resistance to Imatinib (Corbin et al., 2003; Apperley JF 2007). The other reasons for the occurrence of acquired resistance are the clonal evolution that also speeds up the progression to the advanced stage, drug bioavailability, augmentation of the BCR-ABL fusion gene, transporter genes of Imatinib or tyrosine kinases such as Src family kinases being overexpressed, and harmful effects as a result of drug non-compliance or dose reduction (Michele et al., 2006; Sarit et al., 2011; Zaidatul et al., 2014).

\section{Response and Monitoring to TKIs}

European Leukemianet established guidelines for the assessment of the response(Table 1 a) and the time period for the assessment of the response(Table1b) to the TKI therapy. Table 2 defines the category the patient would be placed in according to the time period and the type of response.

Optimal response means continue with the same treatment. Warning means to closely monitor the patient's response. Failure means to switch to another drug. CHRComplete Haematological Response represented by Platelet count $<450 \times 109 / \mathrm{L}$, WBC count $<10 \times 109 / \mathrm{L}$, differential

Table 1 (a). Response to TKI Therapy of Patients According to the Guidelines Provided by the European Leukemianet 2013 (Lahaye et al. 2005; Hughes et al 2006; Hughes 2006; NCCN 2010; Michele et al 2013)

\begin{tabular}{|c|c|c|}
\hline \multirow{2}{*}{ HAEMATOLOGIC RESPONSE } & \multirow{2}{*}{ CYTOGENETIC RESPONSE } & MOLECULAR RESPONSE \\
\hline & & (BCR-ABL1 transcripts) \\
\hline \multicolumn{3}{|l|}{ Complete: } \\
\hline$\checkmark$ Platelet count $<450 \times 10^{9} / \mathrm{L}$ & $\checkmark$ Complete: $\mathrm{Ph}+0 \%$ & $\checkmark$ MR4.5: $\leq 0.003 \%$ \\
\hline$\checkmark$ WBC count $<10 \times 10^{9} / \mathrm{L}$ & $\checkmark$ Partial: $\mathrm{Ph}+1 \%-35 \%$ & $\checkmark$ MR4.0: $\leq 0.01 \%$ \\
\hline $\begin{array}{l}\checkmark \text { Differential without immature granulocytes } \\
\text { and with less than } 5 \% \text { basophils; }\end{array}$ & $\checkmark$ Minor: $\mathrm{Ph}+36 \%-65 \%$ & $\checkmark$ Major3.0: $\leq 0.10 \%$ \\
\hline \multirow[t]{2}{*}{$\checkmark$ Nonpalpable spleen } & $\checkmark$ Minimal: $\mathrm{Ph}+66 \%-95 \%$ & $\checkmark$ Relapse: $>0.5-1.0 \%$ \\
\hline & $\checkmark$ None: $\mathrm{Ph}+>95 \%$ & \\
\hline
\end{tabular}

Major MR3.0 mean at least $3 \log$ reduction in BCR-ABL Transcripts and expressed according to the International Scale

Table 1 (b): Monitoring of TKI Therapy of Patients According to the Guidelines Provided by the European Leukemianet 2013 (Lahaye et al. 2005; Hughes et al 2006; Hughes 2006; NCCN 2010; Michele et al 2013)

\begin{tabular}{|c|c|c|}
\hline $\begin{array}{c}\text { MONITORING OF } \\
\text { HAEMATOLOGICAL RESPONSE }\end{array}$ & $\begin{array}{c}\text { MONITORING OF CYTOGENETIC } \\
\text { RESPONSE }\end{array}$ & $\begin{array}{c}\text { MONITORING OF MOLECULAR } \\
\text { RESPONSE }\end{array}$ \\
\hline Initial: & Initial: & Initial: \\
\hline \multirow[t]{2}{*}{$\begin{array}{l}\text { Every } 2 \text { weeks until complete response } \\
\text { achieved. }\end{array}$} & $\begin{array}{l}\text { Every } 6 \text { months until complete response } \\
\text { achieved. }\end{array}$ & Every 3 months. \\
\hline & & $\begin{array}{l}\text { In case of failure, suboptimal response, } \\
\text { or transcript level increases mutational } \\
\text { analysis required. }\end{array}$ \\
\hline Follow-up: & Follow-up: & \\
\hline Every 3 months & Every 12 months & \\
\hline
\end{tabular}


Table 2. Guidelines for response monitoring according to European Leukemianet 2013(Hughes T et al 2006; Hughes T 2006; NCCN 2010; Michele B et al 2013)

\begin{tabular}{|c|c|c|c|c|}
\hline Response & Time Period & $\begin{array}{l}\text { Haematologic } \\
\text { Response }\end{array}$ & Cytogenetic Response & Molecular Response \\
\hline \multirow{6}{*}{ Optimal Response } & \multirow{2}{*}{03 Months } & \multirow{2}{*}{ CHR } & PCyR & \multirow{2}{*}{ BCR-ABL $1<10 \%$} \\
\hline & & & $(\mathrm{Ph}+: \leq 35 \%$ metaphases $)$ & \\
\hline & \multirow{2}{*}{06 Months } & \multirow{2}{*}{-} & CCyR & \multirow{2}{*}{ BCR-ABL $1<1 \%$} \\
\hline & & & (Ph+:0\% metaphases) & \\
\hline & 12 Months & - & - & BCR-ABL1 $<0.1 \%$ \\
\hline & $\begin{array}{c}>12 \text { Months or any } \\
\text { other time }\end{array}$ & - & - & BCR-ABL1 $\leq 0.1 \%$ \\
\hline \multirow{5}{*}{$\begin{array}{l}\text { Warning/ } \\
\text { Suboptimal } \\
\text { response }\end{array}$} & \multirow{2}{*}{03 Months } & \multirow{2}{*}{-} & $\mathrm{Ph}+:$ & \multirow{2}{*}{ BCR-ABL $1>10 \%$} \\
\hline & & & 36-95\%metaphases & \\
\hline & 06 Months & - & $\mathrm{Ph}+:$ 1-35\%Metaphases & BCR-ABL1 1-10\% \\
\hline & 12 Months & - & - & BCR-ABL1 $>0.1-1 \%$ \\
\hline & $\begin{array}{l}>12 \text { Months or any } \\
\text { other time }\end{array}$ & - & $\mathrm{CCA} / \mathrm{Ph}-(-7$, or $7 \mathrm{q}-)$ & - \\
\hline \multirow{7}{*}{ Failure } & \multirow{2}{*}{03 Months } & \multirow{2}{*}{ Non-CHR } & None: & \multirow{2}{*}{-} \\
\hline & & & (Ph+:>95\%metaphases) & \\
\hline & 06 Months & - & $\mathrm{Ph}+:>35 \%$ metaphases & BCR-ABL $1>10 \%$ \\
\hline & 12 Months & - & $\mathrm{Ph}+:>0 \%$ metaphases & BCR-ABL1 >1\% \\
\hline & \multirow{3}{*}{$\begin{array}{l}>12 \text { Months or any } \\
\text { other time }\end{array}$} & \multirow{3}{*}{ Loss of CHR } & $\sqrt{ }$ Loss of CCyR & \multirow{3}{*}{$\begin{array}{l}\text { Confirmed loss of } \\
\text { MMR* }\end{array}$} \\
\hline & & & $\sqrt{ }$ Mutations & \\
\hline & & & $\checkmark \mathrm{CCA} / \mathrm{Ph}+$ & \\
\hline
\end{tabular}

Optimal response means continue with the same treatment. Warning means to closely monitor the patient's response. Failure means to switch to another drug. CHR-Complete Haematological Response represented by Platelet count <450x109/L, WBC count $<10 x 109 / \mathrm{L}$, differential without immature granulocytes and $<5 \%$ basophils and nonpalpable spleen; PCyR:Partial Cytogenetic Response; CCyR:Complete Cytogenetic Response. Cytogenetic response assessed in 500 interphase cells by Fluorescence in situ hybridization(FISH) or metaphases observed in 20 nuclei. Molecular response is assessed by RT-PCR of the RNA extracted from the blood cells and expressed in ratio of BCR-ABL210/ABL according to the International Scale(IS). CCA/Ph-:clonal chromosome abnormalities in $\mathrm{Ph}-$ cells. $\mathrm{CCA} / \mathrm{Ph}+$ :clonal chromosome abnormalities in $\mathrm{Ph}+$ cells. *:In 2 consecutive tests, of which one with a BCR-ABL1 transcripts level $\geq 1 \%$.

without immature granulocytes and $<5 \%$ basophils and nonpalpable spleen; PCyR, Partial Cytogenetic Response; CCyR, Complete Cytogenetic Response. Cytogenetic response assessed in 500 interphase cells by Fluorescence in situ hybridization (FISH) or metaphases observed in 20 nuclei. Molecular response is assessed by RT-PCR of the RNA extracted from the blood cells and expressed in ratio of BCR-ABL210/ABL according to the International Scale (IS). CCA/Ph-, clonal chromosome abnormalities in Ph- cells. $\mathrm{CCA} / \mathrm{Ph}+$, clonal chromosome abnormalities in $\mathrm{Ph}+$ cells. *, In 2 consecutive tests, of which one with a BCR-ABL1 transcripts level $\geq 1 \%$.

\section{Resistance of CML}

Resistance to imatinib can be labeled as primary or intrinsic when there is no Complete Haematologic Response by 3 months, any cytogenetic response by 6 months, partial cytogenetic response by 12 months or complete cytogenetic response by 18 months. Resistance is referred to as secondary or acquired when there is a loss of previously gained haematological, cytogenetic or molecular response. It also may include the progression to accelerated or blast phase after a period of sustained complete haematological response, occurrence of clonal chromosomal abnormalities, or any other BCR-ABL kinase domain mutations (Hughes et al., 2006; Michele et al., 2006; Simona et al., 2011; Michele et al., 2013; Zafar et al., 2013). Approximately $50 \%$ of the CML patients presenting with either primary or secondary resistance to the Tyrosine Kinase Inhibitors (TKI) therapy have been due to the presence of the BCR-ABL kinase domain mutations (Hochhaus et al., 2002; Lahaye et al., 2005; Branford et al., 2006; Soverini et al., 2006; Jabbour et al., 2006; Sarit et al., 2011).

European Leukemianet (ELN) and European Treatment and Outcome Study gives the following recommendations with regards to the BCR-ABL mutational analysis (Figure 1), (Simona et al., 2011)

Various methods exist for the detection of mutations each having their own specificity and sensitivity. The most common involved are direct sequencing (sensitivity, 15-25\%), sequencing after subcloning of PCR products (sensitivity, 9\%), Denaturing High-Performance Liquid Chromatography (D-HPLC) (sensitivity, 0.1$10 \%)$, pyrosequencing and double gradient denaturing 


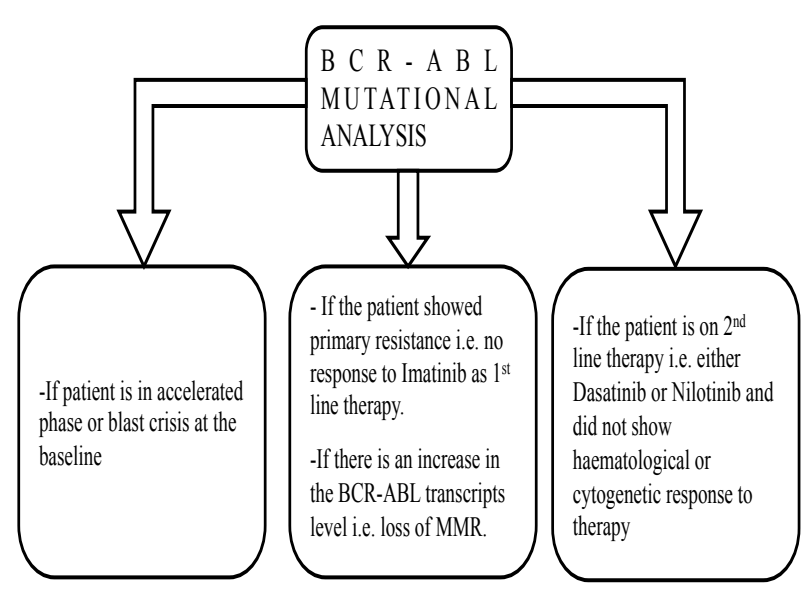

Figure 1. Recommendations for Mutational Analysis electorphoresis (sensitivity, 5\% both). A few other techniques involved in the mutational analysis are Flouorescence PCR and Peptide Nucleic Acid-based Clamping and Allele-Specific Oligonucleotide PCR (ASO-PCR) both with much more better sensitivity (Alderborn et al., 2000; Gorre et al., 2001; Branford et al., 2002; Roche-Lestienne et al., 2002; Shah et al., 2002; Branford et al., 2003; Kreuzer et al., 2003; Roche et al., 2003; Deininger et al., 2004; Irving et al., 2004; Thomas et al., 2005; Sorel et al., 2005; Willis et al., 2005; Khorashad et al., 2006). The method used decides the range of mutations that will be detected. For example, mutations in the BCR-ABL kinase domain will be detected in a patient by direct sequencing only if they are present in greater than 10-20\% of the leukemic cells (Hughes et al., 2006). Caution is thus required at the time of result interpretation. $\mathrm{BCR}-\mathrm{ABL}$ is comprised of four regions that are

Table 3. Functions and Mutations of Regions of BCR-ABL Domain

\begin{tabular}{|c|c|c|c|c|}
\hline REGIONS & IMPORTANCE & MUTATIONS & FREQUENCY & REFERENCES \\
\hline \multirow{6}{*}{ P-Loop } & \multirow{6}{*}{$\begin{array}{l}\text { P-loop repositions } \\
\text { itself in a way that it } \\
\text { folds over the drug } \\
\text { which leads to a much } \\
\text { stronger binding of } \\
\text { the drug to the kinase } \\
\text { domain. }\end{array}$} & $\mathrm{G} 250 \mathrm{E}^{*} / \mathrm{R}$ & & \multirow{6}{*}{$\begin{array}{l}\text { Branford S et } \\
\text { al.2003;Jabbour E et } \\
\text { al.2006; Shundong C } \\
\text { et al. 2008; Ravandi F } \\
2011\end{array}$} \\
\hline & & $\mathrm{E} 255 \mathrm{~K} / \mathrm{V}^{*}$ & 36480 & \\
\hline & & $\mathrm{Q} 252 \mathrm{H}^{*} / \mathrm{R}$ & $30-48 \%$ & \\
\hline & & $\mathrm{Y} 253 \mathrm{H} * / \mathrm{F}$ & & \\
\hline & & M244V & & \\
\hline & & L248V & & \\
\hline
\end{tabular}

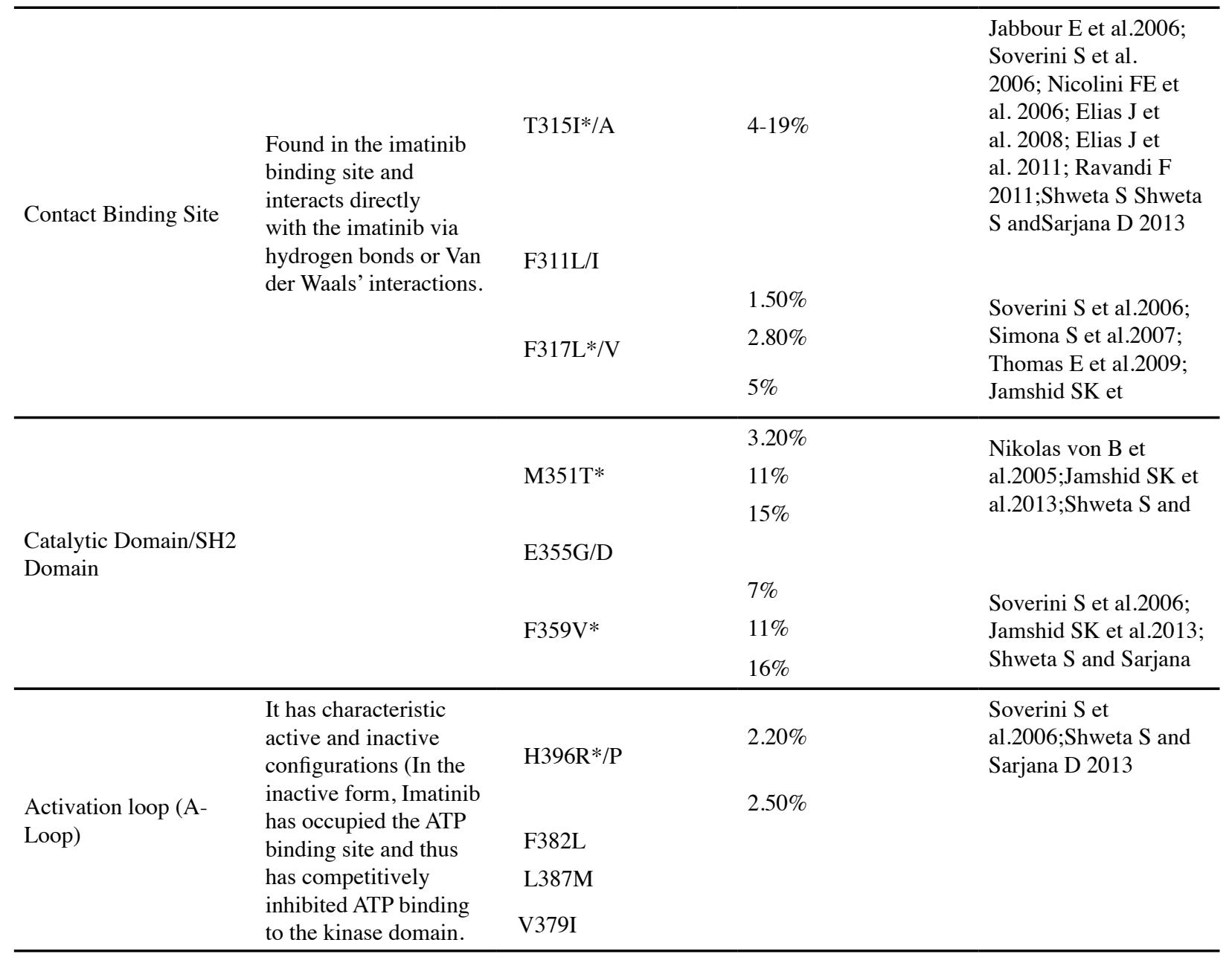

*Most frequent mutations 
composed of vastly preserved series of amino acids and its activity depends on the configuration of these amino acids.

Table 3 gives the function of all the four regions as well as the mutations that have been detected in these specific areas over the years by the employment of various techniques.

The mutations if present may lead to resistance by following mechanisms, (Shah et al., 2000; Apperley, 2007; Elias et al., 2008; Elias et al., 2011),

i) Direct inhibition by creating an alteration in the amino acid that is involved in binding the drug to the kinase for eg. T315I, F317L and F359C/V. ii) Indirect inhibition by creating an alteration in the BCR-ABL conformation for eg. G250E, Q252H, Y253H and E255K/V. iii) One another mechanism leading to resistance due to mutation is the maintenance of the active configuration of BCRABL for eg. M351T and H369R/P.

Of the numerous mutations discovered over the years, the most common ones observed are T315I, M244V, G250E, Y253H, E255K, F317L, M351T, F359V and H396R. The most resilient mutation thus far has been T315I. It accounts for about $4-19 \%$ of all the mutations detected in patients who showed resistance to the TKI therapy and is known as the gatekeeper mutation as it induces resistance by forming a hydrogen bond with the Imatinib through the insertion of the amino acid Isoleucine and thus prevents the binding of the Imatinib to BCRABL sterically (Gorre et al., 2001; Corbin AS et al., 2002; Deininger 2005; Ian et al., 2006; Michael 2006; Elias et al., 2008; Elias et al., 2011). Infact it is the only mutation which shows no response to both the first generation drugs i.e Imatinib as well as the second generation drug i.e , Dasatinib or Nilotinib (Nicolini 2007; Simona et al., 2011). Recent studies have shown the discovery of a pan BCR-ABL inhibitor Ponatinib which seems to overcome the resistance created by the notorious T315I mutation (Zhou et al., 2011; Karunakar et al., 2013). However extensive studies are still required to analyze its effect to gain Complete Cytogenetic Response in patients receiving the drug. Until the time the results get established and the drug receives an approval from FDA, the option available for the patients with T315I mutation seem to be the allogeneic stem cell transplantation (Michele, 2013).

Besides the T315I mutation, the other mutations that are known to confer resistance as well as progression to the advanced stage are mutations lying in the P-Loop. The known mutations of the P-Loop are G250E, Q252H, $\mathrm{Y} 253 \mathrm{~F} / \mathrm{H}, \mathrm{E} 255 \mathrm{~K} / \mathrm{V}$. The P-Loop mutations have been observed to be the most commonly occurring mutations along with T315I (Branford et al., 2003; Jabbour et al., 2006; Shundong and Delong 2008). The P-Loop (A.As, 248-256) is a highly preserved area of the BCR-ABL Kinase domain and is involved in the process of binding of the phosphate group of the ATP (Matti, Peter, Alfred, 1990; Schindler et al., 2000). Studies show that the presence of the P-Loop mutations has been frequently seen in patients who were in the advanced phase of the disease. Also if the patients were found to be in the chronic phase, the presence of the P-Loop mutations eventually led to progression to the advanced stage at a much faster rate as compared to those who did not have these mutations.
DOI:http://dx.doi.org/10.7314/APJCP.2015.16.17.7415

Chronic Myeloid Leukemia - Prognostic Value of Mutations

Thus their presence means a poorer prognosis and early progress to the advanced disease (Branford et al., 2003; Simona et al., 2005; Soverini et al., 2006). Also studies show that the P-Loop mutations are resistant to Nilotinib and so should be given Dasatinib after they have shown no response or failure to Imatinib therapy (Baccarani et al., 2009; Elias et al., 2010; Baccarani et al., 2013; Michele et al., 2013; Michele et al., 2014).

As regards to $\mathrm{F} 317 \mathrm{~L}$ mutation, it has been found that this mutation seems to be resistant to the second generation drug Dasatinib. Patients administered with this drug after no response or failure to Imatinib, showed reduced sensitivity to the drug (Thomas et al., 2009). So patients found with this mutation after the Imatinib failure have been recommended to be treated with another second generation drug, Nilotinib (NCCN 2010; Elias. et al., 2010; Michele et al., 2013; Thoralf. et al., 2013; Michele et al., 2014). M315T lies in the E-helix region and makes contact with the $\mathrm{SH} 2$ domain of the BCR-ABL. This contact leads to a reduced activity of the BCR-ABL kinase activity (Hantschel et al., 2003; Nagar et al., 2003; Nikolas et al., 2005; Willis et al., 2005).

F359V have been found to be resistant to the second generation drug Nilotinib. CML patients found to have this mutation should be given another second generation drug Dasatinib (Baccarani et al., 2009; Baccarani et al., 2013; Michele et al., 2013).

Figure 2 shows the strategic treatment plan for the patients according to their mutational status. Recent studies have indicated that if mutations are detected even at low levels several months before the response is lost, this would mean an eventual resistance and thus a prediction of relapse and progression to the advanced stage (Jamshid et al., 2008; Thomas et al., 2008).

Studies have shown that mutations leading to resistance to the TKIs therapy have much lower incidence in patients in Chronic Phase as compared to those who are in the Accelerated Phase or the Blast crisis (Lahaye et al., 2005; Jabbour et al., 2006; Soverini et al., 2006). Studies also show that mutations present in the Chronic phase leading to the progression to the accelerated phase or the blast crisis is much more frequent than in those patients who had no mutations in the chronic stage of the disease (Shah et al., 2002; Simona et al., 2005; Soverini et al., 2006).

The studies also show that the contribution of the mutations to the occurrence of primary resistance is less in comparison to the acquired one (Lahaye et al., 2005; Jabbour et al., 2006; Soverini et al., 2006). Studies show that the mutations are present in much higher numbers in patients who have been exposed to therapy like interferon and then administered with Imatinib as compared to those who are Imatinib-naïve (Willis et al., 2005; Soverini et al., 2006).

The presence of the mutations in the patients who have not yet been exposed to any therapy and were in the advanced stage disease at the baseline means that the mutations in these people were not due to the exposure to the drug. In fact, the presence of mutations in them is likely to be indicative of some consistent genetic instability as well as the possibility ofworsening of the 


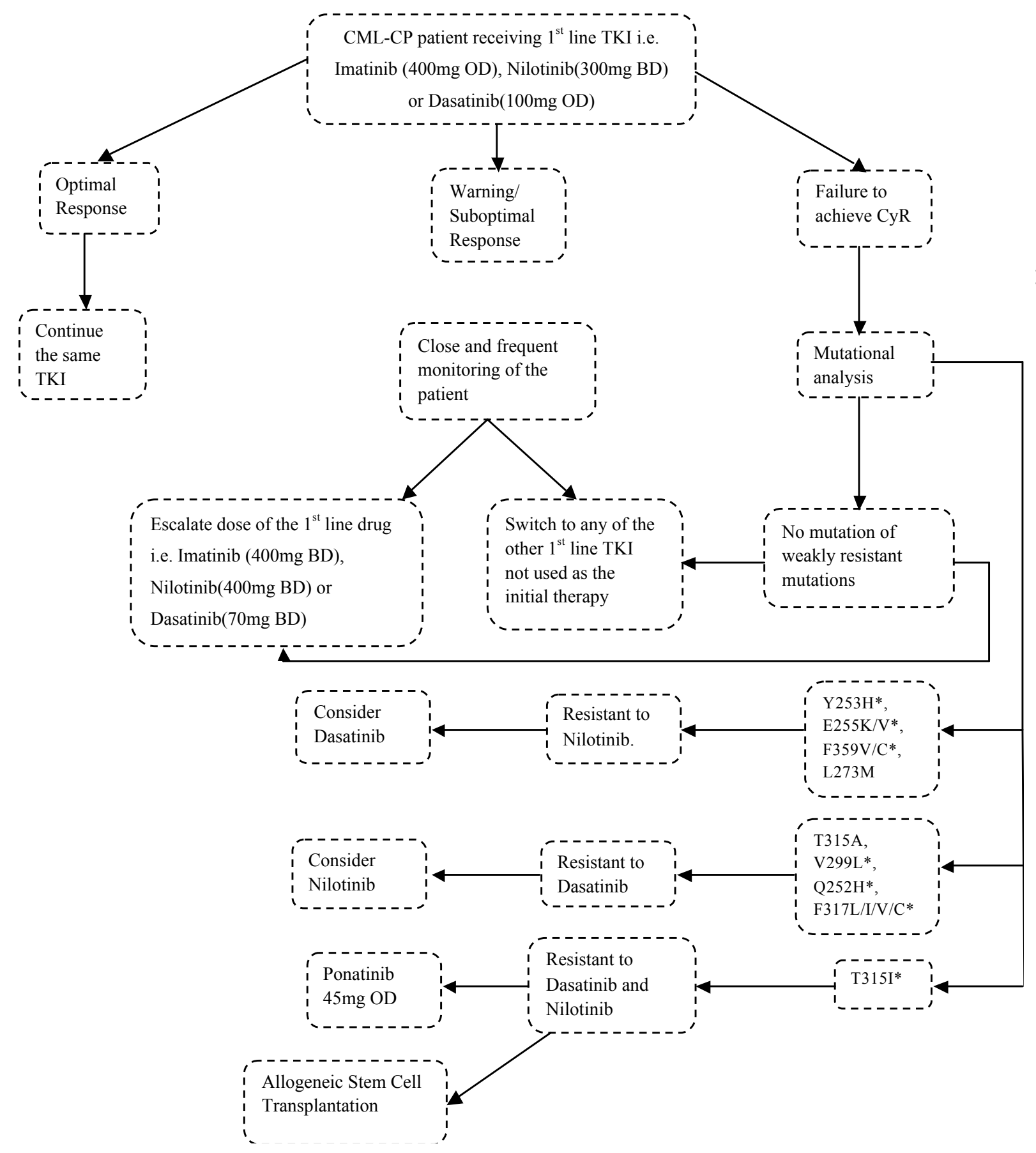

Figure 2. Strategic Treatment Plan for the CML Patients According to the Mutational Status

disease status and thus a poorer outcome (Hughes 2006; Elias et al., 2010).

\section{Conclusions}

The effect of mutations on the disease course has been under investigation for quite a while. European Leukemianet as well as NCCN has provided guidelines regarding the response monitoring of the patients. The algorithm provided by these bodies gives an uncomplicated way of monitoring the response of the patient and tailoring their treatment according to their mutational status. The ultimate goal of all these guidelines is to predict the response a patient might show depending on one's mutational status. Also, besides keeping in view the mutations the patient carries, considerable other factors like patient's Sokal score, his response to the Imatinib should also be kept in mind at the time the patient's future treatment is being decided.

Data have shown that certain mutations show resistance to second generation drug i.e. Dasatinib while another set of mutations are resistant to Nilotinib, another second generation drug. While T315I has shown resistance to both of them as well as the standard treatment i.e. Imatinib. Patients carrying T315I mutation have their question of treatment answered in the form of allogeneic stem cell transplantation or a more recently discovered third generation drug, Ponatinib which is currently under trial 
and is awaiting FDA approval for patient administration. However, overall these set of mutations are less resistant to Dasatinib and Nilotinib as compared to Imatinib. Clinicians have begun to understand the importance of mutational analysis both for the monitoring the response of a certain TKI as well for the decision making at the time of switching to another TKI if the present TKI is not effective. However, extensive trial is in need for further understanding of the importance of the mutations encountered and their eventual significance both in terms of prognosis of the disease as well as the treatment plan.

\section{References}

Alderborn A, Kristofferson A, Hammerling U (2000). Determination of single-nucleotide polymorphisms by real-time pyrophosphate DNA sequencing. Genome Res, 10, 1249-58.

Apperley JF (2007). Part I, mechanisms of resistance to imatinib in chronic myeloid leukaemia. Lancet Oncol, 8, 1018-29.

Branford S, Rudzki Z, Walsh S, et al (2002). High frequency of point mutations clustered within the ATP binding region of BCR/ABL in CML and Ph-positive ALL patients who develop imatinib (STI571) resistance. Blood, 99, 3472-75.

Branford S, Rudzki Z, Walsh S, et al (2003). Detection of BCRABL mutations in patients with CML treated with imatinib is virtually always accompanied by clinical resistance, and mutations in the ATP phosphate-binding loop (P-loop) are associated with a poor prognosis. Blood, 102, 276-83.

Branford S, Hughes T (2006). Detection of BCR-ABL mutations and resistance to imatinib mesylate. Methods $\mathrm{Mol} \mathrm{Med,}$ 125, 93-106.

Baccarani M, Cortes J, Pane F, et al (2009). Chronic myeloid leukemia, an update of concepts and management recommendations of European LeukemiaNet. J Clin Oncol, 27, 6041-51

Björkholm M, Ohm L, Eloranta S, et al (2011). Success story of targeted therapy in chronic myeloid leukemia, a populationbased study of patients diagnosed in Sweden from 1973 to 2008. J Clin Oncol, 29, 2514-20.

Baccarani M, Deininger MW, Rosti G, et al (2013). European LeukemiaNet 2013 recommendations for the management of chronic myeloid leukemia. Blood, 122, 885-92.

Corbin AS, Buchdunger E, Pascal F, Druker BJ (2002). Analysis of the structural basis of specificity of inhibition of the Abl kinase by STI571. J Biol Chem, 277, 32214-19.

Corbin AS, La Rosee P, Stoffregen EP, Druker BJ, Deininger MW (2003). Several Bcr-Abl kinase domain mutants associated with imatinib mesylate resistance remain sensitive to imatinib. Blood, 101, 4611-14.

Deininger MW, McGreevey L, Willis S, et al (2004). Detection of ABL kinase domain mutations with denaturing highperformance liquid chromatography. Leukemia , 18, 864-71.

Deininger M (2005). Resistance to imatinib, mechanisms and management. J Natl Compr Canc Netw. 3, 757-68.

Elias J, Hagop K, Dan J, et al (2008). Characteristics and outcomes of patients with chronic myeloid leukemia and T315I mutation following failure of imatinib mesylate therapy. Blood, 112, 53-5.

Elias J, Andreas H, Jorge C, Paul La R, Hagop MK (2010). Choosing the best treatment strategy for chronic myeloid leukemia patients resistant to imatinib, weighing the efficacy and safety of individual drugs with BCR-ABL mutations and patient history. Leukemia, 24, 6-12.

Elias J, Susan B, Giuseppe S, et al (2011). Practical Advice for Determining the Role of BCR-ABL Mutations in Guiding
DOI:http://dx.doi.org/10.7314/APJCP.2015.16.17.7415 Chronic Myeloid Leukemia - Prognostic Value of Mutations Tyrosine Kinase Inhibitor Therapy in Patients with Chronic Myeloid Leukemia. Cancer, 117, 1800-11.

Fausel C (2007). Targeted chronic myeloid leukemia therapy, Seeking a cure. Am J Health Syst Pharm, 64, 9-15.

Gorre ME, Mohammed M, Ellwood K, et al (2001). Clinical resistance to STI-571 cancer therapy caused by BCR-ABL gene mutation or amplification. Science, 293, 876-80.

Hantschel O, Nagar B, Guettler S, et al (2003). A myristoyl/ phosphotyrosine switch regulates c-Abl. Cell, 112, 845-57.

Gulzar B, Ashaqullah B, Aadil W, et al (2012). Polymorphic variation in glutathione-s-transferase genes and risk of chronic myeloid leukaemia in the kashmiri population. Asian Pac J Cancer Prev, 13, 69-73

Hagop K, Charles S, Andreas H, et al (2002). Hematologic and cytogenetic responses to imatinib mesylate in chronic myelogenous leukemia. $N$ Engl J Med, 346, 645-52.

Hochhaus A, Kreil S, Corbin AS, et al (2002). Molecular and chromosomal mechanisms of resistance to imatinib (STI571) therapy. Leukemia, 16, 2190-96.

Hughes T, Branford S (2006). Molecular monitoring of BCR$\mathrm{ABL}$ as a guide to clinical management in chronic myeloid leukaemia. Blood Rev, 20, 29-41

Hughes T (2006). Abl kinase inhibitor therapy for CML, baseline assessments and response monitoring. Hematol Am Soc Hematol Educ Program, 211-18.

Hughes T, Deininger M, Hochhaus A, et al (2006). Monitoring CML patients responding to treatment with tyrosine kinase inhibitors, review and recommendations for harmonizing current methodology for detecting BCR-ABL transcripts and kinase domain mutations and for expressing results. Blood, 108, 28-37.

Hehlmann R, Hochhaus A, Baccarani M (2007). European LeukemiaNet. Chronic myeloid leukaemia. Lancet, 370, 342-50.

Hochhaus A, Erben P, Ernst T, Mueller MC (2007). Resistance to targeted therapy in chronic myelogenous leukemia. Semin Hematol, 44, 15-24.

Hagop MK, Jorge C, Paul LR, Andreas H (2010). Optimizing therapy for patients with chronic myelogenous leukemia in Chronic Phase. Cancer, 116, 1419-30.

Irving JA, O'Brien S, Lennard AL (2004). Use of denaturing HPLC for detection of mutations in the BCR-ABL kinase domain in patients resistant to imatinib. Clin Chem, 50, 1233-37

Ian JG, Mary MacP, Thomas B (2006). Kinase domain mutants of Bcr-Abl exhibit altered transformation potency, kinase activity, and substrate utilization, irrespective of sensitivity to imatinib. Molecular Cellular Biol, 26, 6082-93

Jabbour E, Kantarjian H, Jones D, et al (2006). Frequency and clinical significance of BCR-ABL mutations in patients with chronic myeloid leukemia treated with imatinib mesylate. Leukemia, 20, 1767-73.

Jamshid SK, Hugues de L, Jane FA, et al (2008). Finding of kinase domain mutations in patients with chronic phase chronic myeloid leukemia responding to imatinib may identify those at high risk of disease progression. J Clin Oncol, 26, 4806-13.

Jamshid SK, Todd WK, Philippe S, et al (2013). BCR-ABL1 compound mutations in tyrosine kinase inhibitor-resistant CML, frequency and clonal relationships. Blood, 121, 489-98.

Kreuzer KA, Le Coutre P, Landt O, et al (2003). Preexistence and evolution of imatinib mesylate-resistant clones in chronic myelogenous leukemia detected by a PNA-based PCR clamping technique. Ann Hematol, 82, 284-96

Khorashad JS, Anand M, Marin D, et al (2006). The presence of a BCR-ABL mutant allele in CML does not always explain 
clinical resistance to imatinib. Leukemia, 20, 658-63.

Kantarjian H, O'Brien S, Jabbour E, et al (2012). Improved survival in chronic myeloid leukemia since the introduction of imatinib therapy, a single-institution historical experience. Blood, 119, 1981-87.

Karunakar T, Lalitha G. Ponatinib (2013). Is a Pan-BCR-ABL Kinase Inhibitor, MD Simulations and SIE Study. Plos One, 8, 1-10.

Lahaye T, Riehm B, Berger U, et al (2005). Response and resistance in 300 patients with BCR-ABL-positive leukemias treated with imatinib in a single center, a 4.5-year follow-up. Cancer, 103, 1659-69.

Matti S, Peter RS, Alfred W (1990). The P-loop --a common motif in ATP- and GTP-binding proteins. Trends Biochem Sci, 15, 430-34.

Michael JM (2006). Defining and managing imatinib resistance. Haematol, 1, 219-25.

Michele B, Giuseppe S, John G, et al (2006). Evolving concepts in the management of chronic myeloid leukemia, recommendations from an expert panel on behalf of the European Leukemianet. Blood, 108, 1809-20.

Meggyesi N, Kozma A, Halm G, et al (2011). Additional chromosome abnormalities, BCR-ABL tyrosine kinase domain mutations and clinical outcome in hungarian tyrosine kinase inhibitor-resistant chronic myelogenous leukemia patients. Acta Haematologica, 127, 34-42

Michele B, Michael WD, Gianantonio R, et al (2013). European LeukemiaNet recommendations for the management of chronic myeloid leukemia, 2013. Blood, 122, 872-84.

Michele B, Fausto C, Gabriele G, Francesca P, Gianantonio R (2014). Treatment Recommendations for Chronic Myeloid Leukemia. Mediterr J Hematol Infect Dis, 6.

Nagar B, Hantschel O, Young MA, et al (2003). Structural basis for the autoinhibition of c-Abl tyrosine kinase. Cell, 112, 859-71

Nikolas von B, Darren RV, Heiko van der K, et al (2005). A cell-based screen for resistance of Bcr-Abl-positive leukemia identifies the mutation pattern for PD166326, an alternative Abl kinase inhibitor. Blood, 105, 1652-59.

Nicolini FE, Corm S, Le QH, et al (2006). Mutation status and clinical outcome of 89 imatinib mesylate-resistant chronic myelogenous leukemia patients, a retrospective analysis from the French intergroup of CML (Fi (phi)-LMC GROUP). Leukemia, 20, 1061-6.

Nicolini FE, Hayette S, Corm S, et al (2007). Clinical outcome of 27 imatinib mesylate-resistant chronic myelogenous leukemia patients harboring a T315I BCR-ABL mutation. Haematologica, 92, 1238-41.

National Comprehensive Cancer Network (NCCN) (2010). Clinical practice guidelines in oncology, chronic myelogenous leukemia. V.2.2010. fort washington, PA, NCCN.

O'Hare T, Eide CA, Deininger MW (2007). Bcr-Abl kinase domain mutations, drug resistance, and the road to a cure for chronic myeloid leukemia. Blood, 110, 2242-9.

Pavlu J, Szydlo RM, Goldman JM, Apperley JF (2011). Three decades of transplantation for chronic myeloid leukemia, what have we learned? Blood, 117, 755-63.

Pavan KB, Hagop K, Jorge EC, A Megan C, Elias J (2013). Management of imatinib-resistant patients with chronic myeloid leukemia. Therapeutic Advances Hematol, 4 , 103-17.

Roche-Lestienne C, Soenen-Cornu V, et al (2002). Several types of mutations of the Abl gene can be found in chronic myeloid leukemia patients resistant to STI571, and they can pre-exist to the onset of treatment. Blood, 100, 1014-8.

Roche-LC, Lai JL, Darre S, Facon T, Preudhomme C (2003). A mutation conferring resistance to imatinib at the time of diagnosis of chronic myelogenous leukemia. $N$ Engl J Med. 348, 2265-6.

Ravandi F (2011). Managing philadelphia chromosome-positive acute lymphoblastic leukemia, role of tyrosine kinase inhibitors. Clin Lymphoma Myeloma Leuk, 11, 198-203.

Schindler T, Bornmann W, Pellicena P (2000). Structural mechanism for STI-571 inhibition of abelson tyrosine kinase. Science, 289, 1938-42.

Shah NP, Nicoll JM, Nagar B, et al (2002). Multiple BCR-ABL kinase domain mutations confer polyclonal resistance to the tyrosine kinase inhibitor Imatinib (STI571) in chronic phase and blast crisis chronic myeloid leukemia. Cancer Cell, 2, 117-25

Sorel N, Chazelas F, Brizard A, Chomel JC (2005). Doublegradient-denaturing-gradient gel electrophoresis for mutation screening of the BCR-ABL tyrosine kinase domain in chronic myeloid leukemia patients. Clin Chem. 51, 1263-66

Simona S, Giovanni M, Gianantonio R, et al (2005). ABL mutations in late chronic phase chronic myeloid leukemia patients with up-front cytogenetic resistance to imatinib are associated with a greater likelihood of progression to blast crisis and shorter survival, a study by the GIMEMA working party on chronic myeloid leukemia. J Clin Oncol, 23, 4100-9.

Soverini S, Colarossi S, Gnani A, et al (2006). Contribution of Abl kinase domain mutations to imatinib resistance in different subsets of Philadelphia-positive patients, by the GIMEMA working party on chronic myeloid leukemia. Clin Cancer Res, 12, 7374-9

Simona S, Sabrina C, Alessandra G, et al (2007). Resistance to dasatinib in Philadelphia-positive leukemia patients and the presence or the selection of mutations at residues 315 and 317 in the BCR-ABL kinase domain. Haematologica 92, 401-4.

Shundong C, Delong L (2008). P-loop mutations and novel therapeutic approaches for imatinib failures in chronic myeloid leukemia. J Hematol Oncol, 1.

Stephen GO'B, François G, John MG, et al (2010). International randomized study of interferon versus STI571 (IRIS) 7-year follow-up, sustained survival, low rate of transformation and increased rate of major molecular response (MMR) in patients with newly diagnosed chronic myeloid leukemia in chronic phase (CML-CP) treated with imatinib (IM). Blood, 116, 3758-65.

Saad ZUsmani, Shakeeb A Yunus, Yasser Jamal (2009). Overview of chronic myeloid leukemia patients in pakistan in the pre-imatanib, Era Asian Pac J Cancer Prev, 10, $1039-40$

Sarit A, Jeffery HL (2011). Monitoring response and resistance to treatment in chronic myeloid leukemia. Current Oncol, 18, $71-83$

Simona.S, Andreas H, Franck EN, et al (2011). BCR-ABL kinase domain mutation analysis in chronic myeloid leukemia patients treated with tyrosine kinase inhibitors, recommendations from an expert panel on behalf of European Leukemia Net. Blood, 118, 1208-15.

Shweta S, Sarjana D (2013). Imatinib mesylate resistance and mutations, An Indian experience. Indian J Med Paediatr Oncol, 34, 213-20

Thomas E, Philipp E, Thomas S, et al (2005). Early detection of imminent hematologic relapse due to BCRABL kinase domain mutations in CML patients on imatinib therapy by D-HPLC. Blood, 106, 317-23

Thomas E, Philipp E, Martin CM, et al (2008). Dynamics of BCR-ABL mutated clones prior to hematologic or cytogenetic resistance to imatinib. Haematologica, 93, 186-92 
Thomas E, Franz X, Oliver PA, et al (2009). A co-operative evaluation of different methods of detecting BCR-ABL kinase domain mutations in patients with chronic myeloid leukemia on second-line dasatinib or nilotinib therapy after failure of imatinib. Haematologica, 94, 1227-35.

Talpaz M, Hehlmann R, Quintás-Cardama A, et al (2013). Re-emergence of interferon- $\alpha$ in the treatment of chronic myeloid leukemia. Leukemia, 27, 803-12

Thoralf L, Thomas E, Franz XG, et al (2013). The quantitative level of T315I mutated BCR-ABL predicts for major molecular response to second-line nilotinib or dasatinib treatment in patients with chronic myeloid leukemia. Haematologica, 98, 714-7.

Willis SG, Lange T, Demehri S, et al (2005). High sensitivity detection of BCR-ABL kinase domain mutations in imatinibnaive patients, correlation with clonal cytogenetic evolution but not response to therapy. Blood, 106, 2128-37.

Yasser HE, Hossam KM, Fahmy TA, et al (2013). Abl Kinase Domain Mutations in Imatinib-treated Egyptian Patients with Chronic Myeloid Leukemia. Leukemia, 1, 1-7.

Yuan-X, Juan Z, Yan-H, et al (2014). Clinical significance of BCR-ABL fusion gene subtypes in chronic myelogenous and acute lymphoblastic leukemias, Asian Pac J Cancer Prev, 15, 9961-6

Zhou T, Commodore L, Huang WS, et al (2011). Structural mechanism of the Pan-BCR-ABL inhibitor ponatinib (AP24534), lessons for overcoming kinase inhibitor resistance. Chem Biol Drug Des, 77, 1-11.

Zafar I, Aamer A, Mudassar I, et al (2013). Sensitive detection of pre-existing BCR-ABL kinase domain mutations in CD34+ cells of newly diagnosed chronic-phase chronic myeloid leukemia patients is associated with imatinib resistance, implications in the post-imatinib Era. Plos One, 8, 1-9.

Zaidatul S, Sarina S, Rosline H, et al (2014). Low level of TERC gene amplification between chronic myeloid leukaemia patients resistant and respond to imatinib mesylate treatment. Asian Pac J Cancer Prev, 15, 1863-9. 